\title{
Visual Analysis of Landscape Aesthetic Evaluation Based on Bibliometric Methods
}

\author{
Qiang Wang ${ }^{1,3, \dagger}$, Mingyuan Zhao ${ }^{2, \dagger}$, Lanyong Zhao ${ }^{2, *}$ \\ ${ }^{1}$ College of Horticultural and Science and Engineering, Shandong Agricultural University, Taian, China \\ ${ }^{2}$ College of Forestry, Shandong Agricultural University, Taian, China \\ ${ }^{3}$ College of Architecture and Landscape Design, Shandong University of Art \& Design, Ji'nan, China
}

\author{
Email address: \\ sdzly369@163.com (Lanyong Zhao) \\ ${ }^{*}$ Corresponding author \\ $\uparrow$ Qiang Wang and Mingyuan Zhao are co-first authors.
}

\section{To cite this article:}

Qiang Wang, Mingyuan Zhao, Lanyong Zhao. Visual Analysis of Landscape Aesthetic Evaluation Based on Bibliometric Methods. American Journal of Civil Engineering. Vol. 9, No. 3, 2021, pp. 63-69. doi: 10.11648/j.ajce.20210903.11

Received: March 6, 2021; Accepted: April 26, 2021; Published: May 8, 2021

\begin{abstract}
In recent decades, landscape aesthetic evaluation has been one of the research hotspots in the field of Landscape Science and Ecology, and Landscape Science, Ecology and other related disciplines have been integrated at multiple levels in the research. In order to sort out and summarize the research process and results in the past decade, with a view to find future research trends, we retrieved and downloaded papers on landscape aesthetic evaluation from the WOS database and analyzed them using a bibliometric method based on mathematical statistics. We used Excel to perform a basic analysis of the growth trends in the number of articles, source journals and countries. Co-citation analysis, Cluster analysis, and Keyword co-occurrence analysis were performed with Citespace, and visual graphs were generated. Based on the combing of landscape aesthetic evaluation research, we analyzed and discussed the research frontier and its evolution, key literature and research hotspots, development trend and knowledge structure in this field, and conclude that: (1) the research in the field in recent decade can be divided into the period of theoretical foundation construction, research system improvement and diversified development, (2) Research methods have developed from mainly evaluating landscape photos to the comprehensive use of GIS, VR, Eye-tracking, EEG testing and other technological means, and (3) research hotspots have expanded from Ecology, Scenic beauty evaluation and Landscape management to Rural landscape, Urban public space and Land use, representing the future development trend.
\end{abstract}

Keywords: Landscape Aesthetic Evaluation, Citespace, Web of Science, Bibliometric Methods

\section{Introduction}

Since the 1960s, landscape aesthetic (preference) evaluation has been one of the research hotspots in the field of Landscape Science and Ecology, and this research direction involves disciplines such as Landscape Science, Ecology, Aesthetics, Psychology, Geography, etc. It crosses multiple lines between these disciplines, spanning both the natural and social science. Many scholars have been trying to study the proposition of aesthetic evaluation of landscape, which is inclined to social science, with the help of natural science research methods (mathematical statistics, etc.), and have obtained important research results [1-7]. For the most part, these studies are included in the framework of research on cultural ecosystem services, and the general consensus that can be obtained through these studies is that landscape aesthetics is an important part of cultural ecosystems and that its contribution to human well-being is widely recognized. However, because cultural ecosystem services involve multiple disciplines and a wide range of research methods, a satisfactory understanding of many important aspects has yet to be reached. Our search from the WOS platform found only about 12 review papers on landscape aesthetic evaluation research, of which the highly cited paper is Cultural Ecosystem Services: A Literature Review and Prospects for Future Research. The authors reviewed 107 publications and grouped them into studies, and the results suggest that developing consistent approaches to the study of cultural 
ecosystem services is a particular challenge for researchers, and that these approaches may be useful in the future to address practical issues in the management of human-nature interactions $[8,9]$.

In order to summarize the process and results of research in the field of landscape aesthetic evaluation with a view to making new discoveries, this paper bypasses the traditional descriptive qualitative analysis method and uses a quantitative bibliometric method based on mathematical statistics to visually analyze the representative literature data in this direction in the last decade or so, hoping to reveal the connection patterns between the data precisely and intuitively. Specifically, we used the core database of the WOS platform (Web of Science Core Collection) to search the papers on aesthetic evaluation of landscape since 2011 and conduct visual mathematical and statistical analysis (Excel, Citespace) to explore the historical evolution, research hotspots and future research trends of this field in a certain period (2011-2020).

\section{Methodology}

\subsection{Literature Sources and Search}

Literature source: Web of Science Core Collection

Search method: The choice of search method needs to be balanced with the comprehensiveness and accuracy of the search, because the term "landscape" in English is used in a wide range of research fields, including environmental science, ecology, geography, medicine, zoology, chemistry, etc., such as the use of a single search method of subject search will lead to a low search accuracy rate. After analyzing and comparing the comprehensiveness and accuracy of the results generated by different search methods, we finally adopted a combined search method. The core term of the search is landscape, and the second is visual or aesthetics or preference. Title search (TI) and keywords are used respectively, and the search document type is "Article", the language is English, and then the retrieval results are combined to retrieve a total of 580 English papers (Table 1). After screening and eliminating papers in unrelated research fields such as medicine, zoology, chemistry, etc., 412 academic papers in the search results are retained for analysis. The search results are exported in plain text, saving information such as title, abstract, keywords, and references.

Table 1. Search strategy and results.

\begin{tabular}{ll}
\hline Search strategy $($ TI=Title, AK= Author Keywords) & Results \\
\hline $1 \#:$ TI $=(($ visual* or aesthetic* or preference $)$ AND landscape $)$ & 348 \\
2\#: AK = ((visual* or aesthetic* or preference) AND landscape) & 385 \\
1\# OR 2\# & 580 \\
Refine & 412 \\
\hline
\end{tabular}

\subsection{Analysis Method}

This paper focuses on literature co-citation and keyword co-occurrence analysis using Citespace and Co-citation relationships in the literature reflect the connections between key concepts, which is a way to reflect the structure of knowledge in the discipline. Small H (1973), one of the originators of this concept, gradually refined and validated the co-citation method by mapping the knowledge structure of many fields in natural and social sciences [10], and used the method to detect research frontiers, which is characterized by the rapid growth of research results and innovative content, as well as a certain scale of research.

In searching for emerging concepts, Citespace used the Burst Dection Algorithum of Kleinberg, J (2002), which indicates research frontiers by finding Burst Terms with dramatic changes in frequency from the cited literature [11].

\section{Results}

\subsection{Excel Basic Analysis}

\subsubsection{Publication Years}

From 2011 to 2020, the number of papers published in landscape aesthetic evaluation showed a flat growth trend, increasing from 22 in 2011 to 55 in 2020, with an average annual growth rate of $10.71 \%$, including an accelerated growth rate of $20.08 \%$ in 2017-2019 (Figure 1).

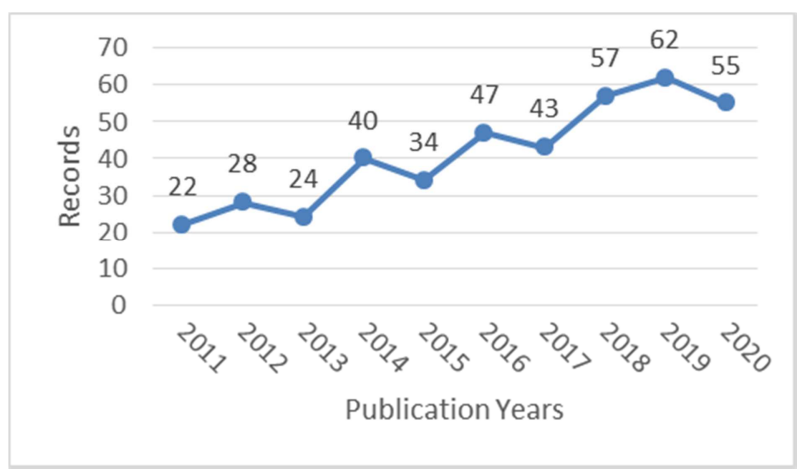

Figure 1. Number of records.

\subsubsection{Source Titles}

There are 8 journals with more than 10 articles published in the following table (Table 2), including 64 articles in LANDSCAPE AND URBAN PLANNING, which was founded in 1974 as Landscape Planning, a monthly academic journal published by Elsevier. It merged with Urban Ecology in 1986, and changed to its current name. The journal covers the fields of landscape science (including landscape planning, design and architecture), urban and regional planning, landscape and ecological engineering, and landscape and urban ecology.

\subsubsection{Countries or Regions}

From the source countries/regions of the articles, the top ten countries and regions are mainly from Europe, America and China, among which the United States (89) and China (67) have the largest number of articles, and the sum of the two accounts for more than $37 \%$ of the total number of articles, occupying the mainstream position of landscape aesthetic evaluation research (Figure 2). 
Table 2. Main source titles.

\begin{tabular}{lll}
\hline Source Titles & records & \% of 412 \\
\hline LANDSCAPE AND URBAN PLANNING & 64 & 15.53 \\
SUSTAINABILITY & 31 & 7.52 \\
URBAN FORESTRY URBAN GREENING & 31 & 7.52 \\
FRESENIUS ENVIRONMENTAL BULLETIN & 13 & 3.16 \\
ECOLOGICAL INDICATORS & 12 & 2.91 \\
LANDSCAPE ECOLOGY & 12 & 2.91 \\
JOURNAL OF ENVIRONMENTAL & 11 & 2.67 \\
MANAGEMENT & 10 & 2.43 \\
ECOSYSTEM SERVICES & & \\
\hline
\end{tabular}

Figure 2. Countries or Regions.

\subsection{Citespace Analysis}

\subsubsection{Co-citation Analysis}

We imported 412 English sample data searched from WOS into Citespace, with the time interval from 2011 to 2020, Time Slice selected 1, divided into 10 time slices, and the top 50 cited records were filtered in each time slice (Top $\mathrm{N}=50$ ), Node Type selected Reference, and other parameters kept as

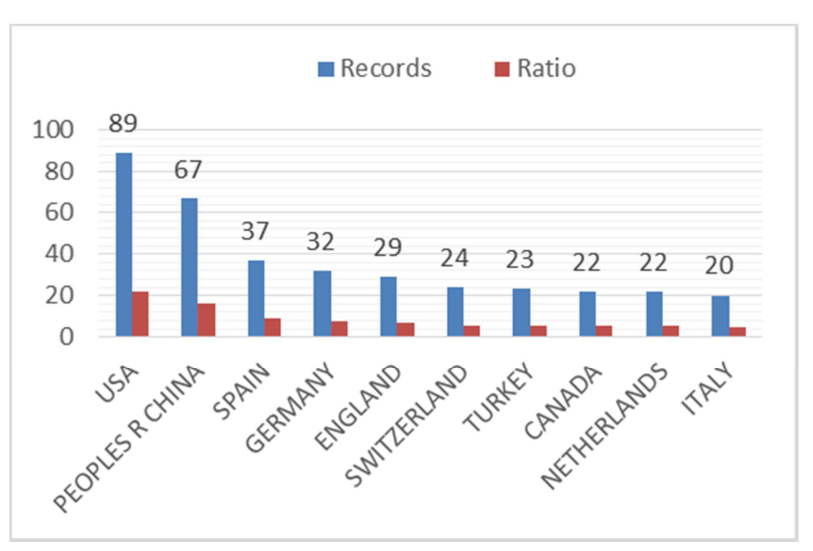

default.

After analysis by Citespace, 410 valid literature data were obtained, and the total number of cited references was 16239 , and the literature co-citation network had 323 nodes and 1307 links. After further cluster analysis, a total of 33 co-citation clusters were generated, with a cluster module value (Modularity) of 0.6572 (greater than 0.3 was considered significant for cluster structure) and a cluster mean silhouette value (Mean Silhouette) of 0.8779 (greater than 0.5 was considered reasonable for clustering and greater than 0.7 was considered convincing), as shown in Figure 3, Figure 4, and Table 3 .

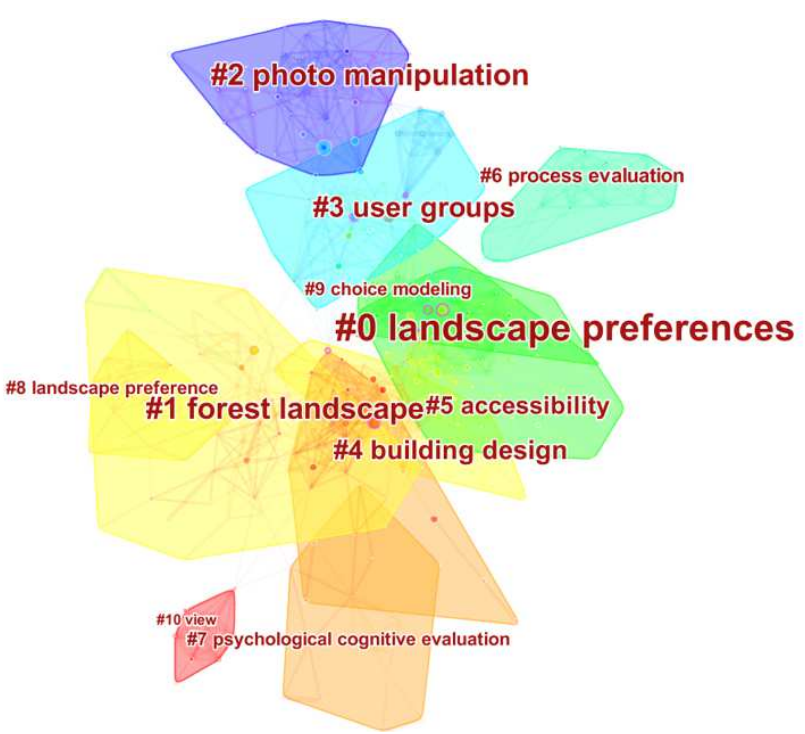

Figure 3. Top ten cluster view.

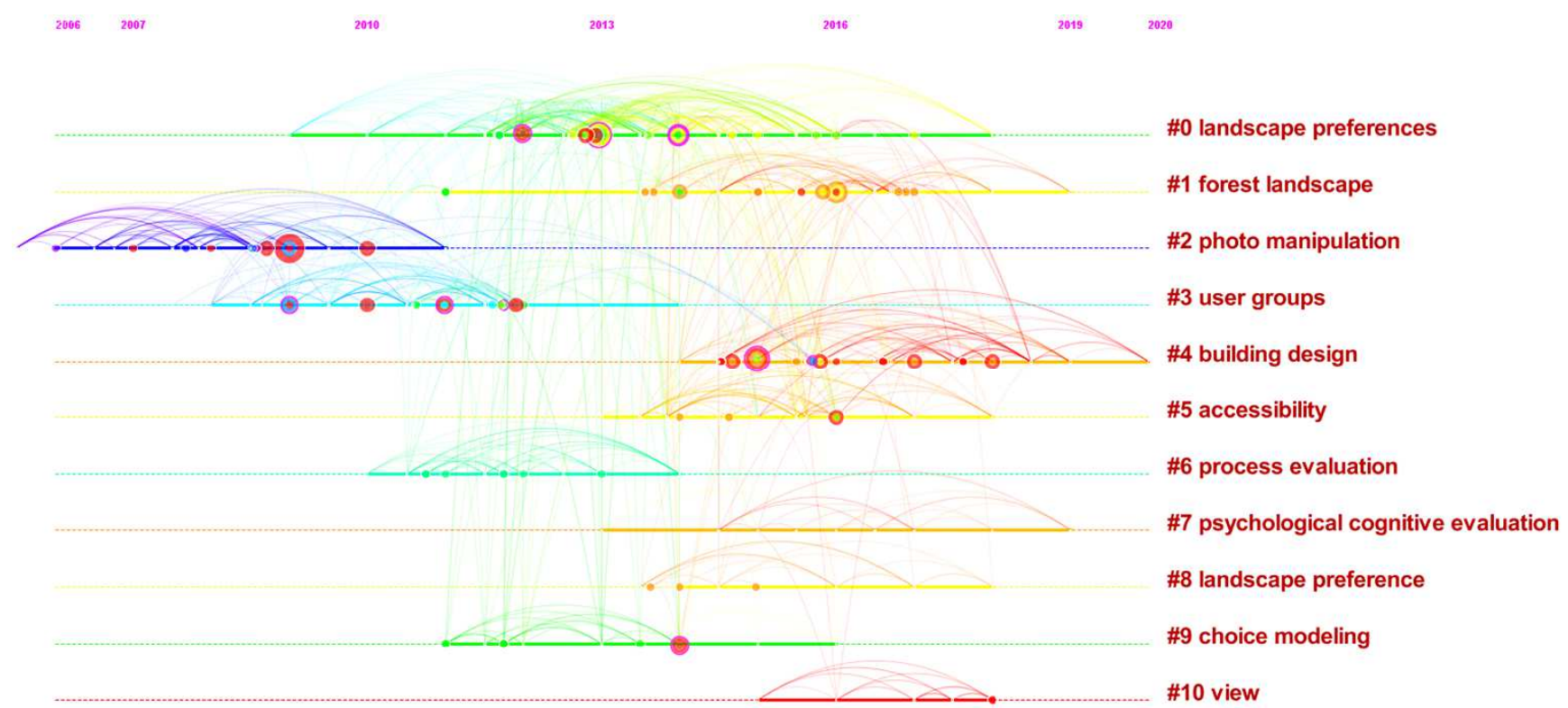

Figure 4. Top ten cluster timeline view. 
Table 3. Top ten cluster information.

\begin{tabular}{lllll}
\hline Cluster ID & Size & Silhouette & Mean (Year) & Top Terms (LLR) \\
\hline 0 & 57 & 0.836 & 2013 & landscape preferences; cultural ecosystem services; social perceptions \\
1 & 40 & 0.804 & 2015 & forest landscape; visual aesthetic quality; landscape preferences \\
2 & 39 & 0.909 & 2008 & photo manipulation; visualization tools; landscape fuzziness \\
3 & 34 & 0.893 & 2010 & user groups; choice modelling; landscape features \\
4 & 30 & 0.87 & 2016 & building design; visual comfort; image content analysis \\
5 & 25 & 0.772 & 2015 & accessibility; conflict assessment; land use change \\
6 & 17 & 0.994 & 2011 & process evaluation; landscape visualization; policy outcomes \\
7 & 14 & 0.975 & 2016 & psychological cognitive evaluation; scenario visualization; forest landscape space \\
8 & 14 & 0.992 & 2015 & landscape preference; stormwater management; preference \\
9 & 14 & 0.916 & 2012 & choice modeling; mapping; agricultural landscape \\
10 & 9 & 0.991 & 2017 & view; urban; mental health \\
\hline
\end{tabular}

\subsubsection{Cluster Analysis}

As seen from the clustering timeline view (Figure 4), the landscape aesthetic preference studies in the last decade are roughly divided into three stages based on the average publication time of the references. The first stage, from 2008 to 2010 , contains clusters $2 \#$ and $3 \#$, is characterized by the construction of basic theories and research systems; the second stage, from 2011 to 2013 , contains clusters 0\#, 6\#, and $9 \#$, is characterized by a period of perfect development of research systems; the third stage, from 2015 to 2017, contains clusters $1 \#, 4 \#, 5 \#, 7 \#, 8 \#$, and $10 \#$, is characterized by multiple development.

The first stage contains clusters $2 \#$ and $3 \#$. The $2 \#$ cluster named photo manipulation contains top terms such as visualization tools, landscape fuzziness, landscape preferences, photo based questions, etc., and main papers such as Indicators of perceived naturalness as drivers of landscape preference [1], The use of latent classes to identify individual differences in the importance of landscape dimensions for aesthetic preference [12], Indicators of visual scale as predictors of landscape preference; a comparison between groups [13], it can be seen that early landscape aesthetic evaluation research focused on basic theoretical research and the construction of research method systems, such as selecting appropriate evaluation indicators in order to establish a research system from abstract visual concepts to measurable visual indicators, and using landscape photographs as the main carrier of landscape evaluation.

The literature focused on cluster $3 \#$ mostly takes rural landscape evaluation as a research vehicle and investigates the differences in landscape aesthetic evaluation among different types of groups, such as Landscape aesthetics: Assessing the general publics' preferences towards rural landscapes [2], Aesthetic preference for a Swiss alpine landscape: The impact of different agricultural land-use with different biodiversity [14], Cognitive attributes and aesthetic preferences in assessment and differentiation of landscapes [15].

The second stage contains clusters \#0, \#6 and \#9. Cluster 0\#, labeled landscape preferences, is the largest cluster, containing 57 articles with top terms including cultural ecosystem services, social perceptions, and drylands. Articles in this cluster consider landscape as an important part of cultural ecological services, and proposed methods to quantify them, such as Spatial quantification and valuation of cultural ecosystem services in an agricultural landscape [16], while incorporating techniques such as GIS into the aesthetic evaluation of landscapes, such as Predicting scenic beauty of mountain regions [17].

The label for cluster \#6 is process evaluation, Its top terms include landscape visualization, policy outcomes, virtual globe, participatory decision-making. The main components of the articles in this cluster is landscape visualization, such as Identifying strengths and weaknesses of landscape visualization for effective communication of future alternatives [18], Which is the Appropriate 3D Visualization Type for Participatory Landscape Planning Workshops? A Portfolio of Their Effectiveness [19].

Cluster \#9 which labeled Choice Modeling, focus on agricultural landscapes and Ecosystem service assessments (ESA), its top terms include mapping, agricultural landscape, comparative study, landscape aesthetics. The key article is Preferences for European agrarian landscapes: A meta-analysis of case studies [20], Using meta-analysis methods to compare agricultural landscape preferences in a series of European case studies, the authors found that general preferences for livestock, mosaic land and historic buildings, as well as landscape attributes preferences were associated with population density and income.

The third stage contains clusters $1 \#, 4 \#, 5 \#, 7 \#, 8 \#$, and $10 \#$, and the average year of publication for the literature is 2015-2017. Cluster 1\# is labeled Forest Landscape, and its top terms include visual aesthetic quality, landscape preference, landscape metrics. VAQ (visual aesthetic quality) in green space is the main focus of articles within this cluster, such as Consensus in visual preferences: The effects of aesthetic quality and landscape types [21], Demographic groups' differences in visual preference for vegetated landscapes in urban green space [22], Visual landscape preferences in Mediterranean areas and their socio-demographic influences [23].

Cluster 4\#, labeled building design, includes top terms such as visual comfort, image content analysis, visual assessments. The articles in this cluster reflect the diversification of research methods for landscape aesthetic evaluation in recent years, such as the collection and analysis of social media data $[24,25]$, the use of digital models of the landscape [26], and analysis based on eye-tracking [27].

Cluster 5\# is named accessibility and it mainly studies landscape aesthetic evaluation based on cultural ecosystem 
services. Different from cluster 0\#, it emphasizes the evaluation of cultural ecosystem services (landscape aesthetics) from the perspective of social culture rather than monetary value, which shows the further development of the research. Such as Integrating socio-cultural perspectives into ecosystem service valuation: A review of concepts and methods [28].

Cluster 7\# (Psychological Cognitive Evaluation) focuses on the visual and psychological perception of environmental elements in the aesthetic evaluation of the landscape, such as Investigating the visual exploration of the rural-urban gradient using eye-tracking [29].

Cluster 8\# which labeled landscape preference focuses on restorative environment. For example, Evaluating restoration in urban green spaces: Does setting type make a difference? [30], It argues that restoration in urban public spaces depends on the perceptions and needs of individuals and the physical characteristics of the environment through an experimental comparison of the restorative impacts of urban streetscapes and three common urban park woodland environments. Another paper, Nature and Health-Annual Review of Public Health [31], focused on the health benefits of contact with natural landscapes through a variety of pathways, such as air quality, physical activity, social cohesion, and stress reduction.

The label for cluster 10\# is view, Its top terms include urban; mental health; contemplative landscape etc. (Table 1). Papers in the cluster were mainly related to the relationship between nature or contemplative landscape and mental health. Such as Toward dynamic urban environmental exposure assessments in mental health research [32]. This paper analyzes the differences between static and dynamic exposure assessments in urban environments and proposes a method for assessing the impact of dynamic exposure in urban environments on human mental health.

\subsection{Keyword co-occurrence Analysis}

The keywords of an article are the author's summary and refinement of the article content and express the theme of the article. Keyword co-occurrence analysis is a type of co-word analysis which was developed in the 1970s. Its main principle is to pair a set of words two by two and count their occurrences in the same document. Keyword co-occurrence analysis uses words as units and shows the relationship between concepts [33]. Therefore, the co-occurrence analysis of keywords can get the research hotspots and research trends in a certain period. We import the title, keywords, abstracts and other information of 412 articles into CiteSpace software for keyword co-occurrence analysis. We can get the keyword co-occurrence view, high-frequency keyword list and burst keyword list (Figures 5, 6).

\subsubsection{Keyword co-occurrence}

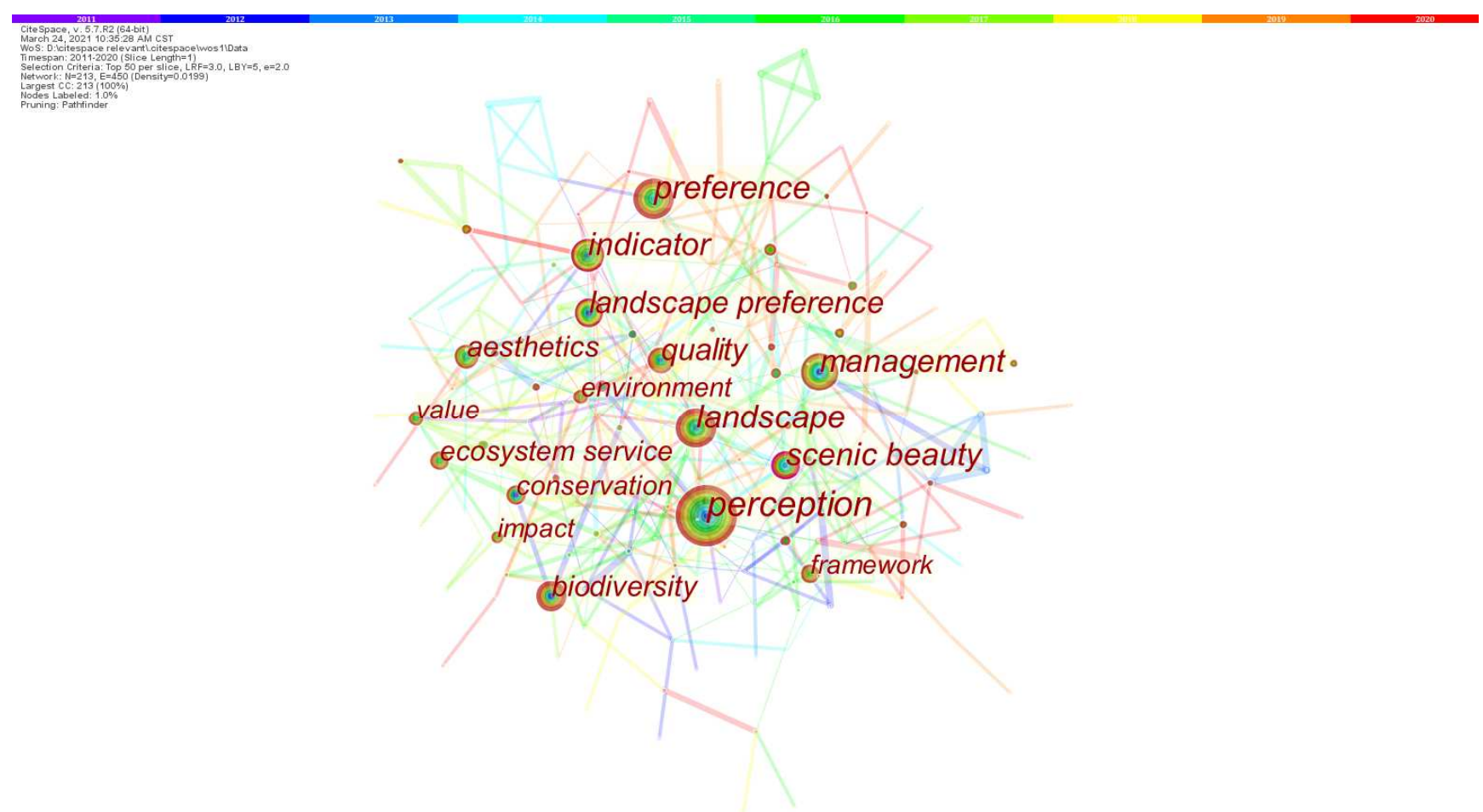

Figure 5. Keyword co-occurrence view.

The keyword co-occurrence chart (Figure 5) shows that, excluding landscape, preference, and aesthetic* included in the search formula, the top six keywords in terms of frequency are perception, indicator, management, scenic beauty, quality, and biodiversity. We can find the research themes and hot spots of visual aesthetic evaluation of landscape in the last 
decade.

\section{Top 11 Keywords with the Strongest Citation Bursts}

\begin{tabular}{|c|c|c|}
\hline \multirow{2}{*}{$\begin{array}{c}\text { Keywords } \\
\text { landscape preference }\end{array}$} & \multicolumn{2}{|c|}{ Year Strength Begin End 2011 - 2020} \\
\hline & 2011 & $3.8920112013=$ \\
\hline attitude & 2011 & 2.8320112014 \\
\hline conservation & 2011 & 2.520112014 \\
\hline scenic beauty & 2011 & 5.1120122015 \\
\hline visualization & 2011 & 2.4820152015 \\
\hline rural landscape & 2011 & 4.0420162017 \\
\hline model & 2011 & 3.220162017 \\
\hline urban & 2011 & 3.2620192020 \\
\hline green space & 2011 & 2.6420192020 \\
\hline urbanization & 2011 & 2.5420192020 \\
\hline land use change & 2011 & 2.5220192020 \\
\hline
\end{tabular}

Figure 6. Keyword bursts.

\subsubsection{Keyword Bursts Analysis}

Burst detection reflects a sudden increase in the frequency of keywords in a certain period. We can use the Cite Space software to find out the bursting hotspots and their durations in each time period. From Figure 6, we can see that the latest burst keywords are Urban, Green Space, Urbanization, and Land Use Change, which represent the research frontiers and hot spots in this field in recent years, and the top three burst keywords are Scenic Beauty (5.11), Rural Landscape (4.04), and Landscape Preference (3.89), among which Rural Landscape1 has a high burst intensity, but the burst period is only 2 years (2016-2017), representing its high research intensity and short duration. The keywords with higher burst intensity and frequency can reflect some research hotspots more comprehensively, for example, the burst period of Scenic Beauty is 2012-2015, and the burst intensity $(5.11,1 \mathrm{st})$ and total citation frequency $(59,5$ th $)$ are both higher, reflecting that this topic was widely followed and studied and became a hotspot in 2012-2015.

\section{Conclusion}

The above analysis of visual co-citation of landscape aesthetic evaluation literature can lead to the following conclusions.

1. The development of landscape aesthetic evaluation in the past decade has experienced a progressive development process from basic theory exploration, research method system construction and improvement, and continuous expansion of the scope of research objects.

2 . The continuous development of research methods and research equipment has promoted the depth of research in this field, such as from the early use of black-and-white slides as the evaluation medium to the application of VR, GIS, Eye-tracking [34], EEG testing and other comprehensive means that have been widely used in recent years.

3. In the keyword co-occurrence analysis, we found that landscape aesthetic evaluation gradually expanded from the concepts of ecology, beauty degree analysis, and landscape management in the early stage to rural landscape, urban public space, and land use in recent years, reflecting the evolutionary trend of research hotspots.

\section{References}

[1] Å. Ode, G. Fry, MS. Tveit, P. Messager, D. Miller, Indicators of perceived naturalness as drivers of landscape preference, Journal of Environmental Management, Volume 90, Issue 1, 2009, Pages 375-383.

[2] P. Howley, Landscape aesthetics: Assessing the general publics' preferences towards rural landscapes, Ecological Economics, Volume 72, 2011, Pages 161-169.

[3] R. B. Hull, W. P. Stewart, Validity of photo-based scenic beauty judgments, Journal of Environmental Psychology, 12 (1992), pp. 101-114.

[4] T. C. Daniel, R. S. Boster, Measuring Landscape Aesthetics: the Scenic Beauty Estimation Method, Journal of Environmental Psychology, 12 (1992), pp. 101-114.

[5] Oteros-Rozas, E., et al., Using social media photos to explore the relation between cultural ecosystem services and landscape features across five European sites, Ecological Indicators, 2018, 94: 74-86.

[6] Tieskens, K. F., et al., Aesthetic appreciation of the cultural landscape through social media: An analysis of revealed preference in the Dutch river landscape, Landscape and Urban Planning, 2018, 177: 128-137.

[7] Townsend, J. B. and S. Barton, The impact of ancient tree form on modern landscape preferences, Urban Forestry \& Urban Greening, 2018, 34: 205-216.

[8] Tribot, A. S., et al., Integrating the aesthetic value of landscapes and biological diversity, Proceedings of the Royal Society B-Biological Sciences, 2018, 285 (1886): 10.

[9] Milcu, A. I., et al., Cultural Ecosystem Services: A Literature Review and Prospects for Future Research, Ecology and Society, 2013, 18 (3): 34.

[10] E. Garfield, Citation indexing: Its theory and application in science, technology, and humanities. Information Sciences Series. New York: John Wiley \& Sons, 1979.

[11] Chen. C. Cite Space II: Detecting and visualizing emerging trends and transient patterns in scientific literature [J]. Journal of the Association for Information Science \& Technology, 57 (03), 2014: 359-377.

[12] Marjanne Sevenant, Marc Antrop, The use of latent classes to identify individual differences in the importance of landscape dimensions for aesthetic preference, Land Use Policy, Volume 27, Issue 3, 2010, Pages 827-842.

[13] Mari Sundli Tveit, Indicators of visual scale as predictors of landscape preference; a comparison between groups, Journal of Environmental Management, Volume 90, Issue 9, 2009, Pages 2882-2888.

[14] Petra Lindemann-Matthies, Reinhold Briegel, Beatrice Schüpbach, Xenia Junge, Aesthetic preference for a Swiss alpine landscape: The impact of different agricultural land-use with different biodiversity, Landscape and Urban Planning, Volume 98, Issue 2, 2010, Pages 99-109.

[15] Marjanne Sevenant, Marc Antrop, Cognitive attributes and aesthetic preferences in assessment and differentiation of landscapes, Journal of Environmental Management, Volume 90, Issue 9, 2009, Pages 2889-2899. 
[16] Derek B. van Berkel, Peter H. Verburg, Spatial quantification and valuation of cultural ecosystem services in an agricultural landscape, Ecological Indicators, Volume 37, Part A, 2014, Pages 163-174.

[17] Uta Schirpke, Erich Tasser, Ulrike Tappeiner, Predicting scenic beauty of mountain regions, Landscape and Urban Planning, Volume 111, 2013, Pages 1-12, ISSN 0169-2046.

[18] Christopher J. Pettit, Christopher M. Raymond, Brett A. Bryan, Hayden Lewis, Identifying strengths and weaknesses of landscape visualisation for effective communication of future alternatives, Landscape and Urban Planning, Volume 100, Issue 3, 2011, Pages 231-241, ISSN 0169-2046.

[19] Hayek, U. W, Which is the Appropriate 3D Visualization Type for Participatory Landscape Planning Workshops? A Portfolio of Their Effectiveness, 2011, 38 (5): 921-939.

[20] van Zanten, B. T., Verburg, P. H., Espinosa, M. et al, European agricultural landscapes, common agricultural policy and ecosystem services: a review, Agron. Sustain, Dev. 34 (2014), 309-325.

[21] Ronghua Wang, Jingwei Zhao, Zhenyu Liu, Consensus in visual preferences: The effects of aesthetic quality and landscape types, Urban Forestry \& Urban Greening, Volume 20, 2016, Pages 210-217.

[22] Ronghua Wang, Jingwei Zhao, Demographic groups' differences in visual preference for vegetated landscapes in urban green space, Sustainable Cities and Society, Volume 28, 2017, Pages 350-357.

[23] Francisco López-Martínez, Visual landscape preferences in Mediterranean areas and their socio-demographic influences, Ecological Engineering, Volume 104, Part A, 2017, Pages 205-215.

[24] Koen F. Tieskens, Boris T. Van Zanten, Catharina J. E. Schulp, Peter H. Verburg, Aesthetic appreciation of the cultural landscape through social media: An analysis of revealed preference in the Dutch river landscape, Landscape and Urban Planning, Volume 177, 2018, Pages 128-137.
[25] van Zanten, B. T., et al., Continental-scale quantification of landscape values using social media data, Proceedings of the National Academy of Sciences, 2016, 113 (46): 12974.

[26] Rob Kuper, Evaluations of landscape preference, complexity, and coherence for designed digital landscape models, Landscape and Urban Planning, Volume 157, 2017, Pages 407-421.

[27] Lien Dupont, Kristien Ooms, Marc Antrop, Veerle Van Eetvelde, Comparing saliency maps and eye-tracking focus maps: The potential use in visual impact assessment based on landscape photographs, Landscape and Urban Planning, Volume 148, 2016, Pages 17-26.

[28] Samantha S. K. Scholte, Astrid J. A. van Teeffelen, Peter H. Verburg, Integrating socio-cultural perspectives into ecosystem service valuation: A review of concepts and methods, Ecological Economics, Volume 114, 2015, Pages 67-78.

[29] Dupont, L., et al., Investigating the visual exploration of the rural-urban gradient using eye-tracking, Spatial Cognition \& Computation, 2017, 17 (1-2): 65-88.

[30] Agnes E. Van den Berg, Anna Jorgensen, Edward R. Wilson, Evaluating restoration in urban green spaces: Does setting type make a difference?, Landscape and Urban Planning, Volume 127, 2014, Pages 173-181.

[31] Hartig, T., et al., Nature and Health, 2014, 35 (1): 207-228.

[32] Marco Helbich, Toward dynamic urban environmental exposure assessments in mental health research, Environmental Research, Volume 161, 2018, Pages 129-135.

[33] Qiu. J. P, Information Management Science, Wuhan University Press: Wuhan, China, 2007.

[34] Lien Dupont, Marc Antrop \&Veerle Van Eetvelde. Eye-tracking Analysis in Landscape Perception Research: Influence of Photograph Properties and Landscape Characteristics [J]. Landscape Research Volume 39, 2014: 417-432. 